\title{
Coronavirus disease (COVID-19) and the liver: a comprehensive systematic review and meta-analysis
}

\author{
Praveen Kumar-M ${ }^{1}$. Shubhra Mishra ${ }^{2}$ Daya Krishna Jha ${ }^{2} \cdot$ Jayendra Shukla ${ }^{2} \cdot$ Arup Choudhury $^{2} \cdot$ Ritin Mohindra $^{3}$.

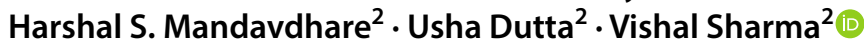

Received: 8 May 2020 / Accepted: 25 June 2020 / Published online: 4 July 2020

(c) Asian Pacific Association for the Study of the Liver 2020

\begin{abstract}
Background Liver function derangements have been reported in coronavirus disease (COVID-19), but reported rates are variable.

Methods We searched PubMed and Embase with terms COVID and SARS-COV-2 from December 1, 2019 till April 5, 2020. We estimated overall prevalence, stratified prevalence based on severity, estimated risk ratio (RR), and estimated standardized mean difference (SMD) of liver function parameters in severe as compared to non-severe COVID. Random effect method utilizing inverse variance approach was used for pooling the data.

Results In all, 128 studies were included. The most frequent abnormalities were hypoalbuminemia [61.27\% (48.24-72.87)], elevations of gamma-glutamyl transferase (GGT) [27.94\% (18.22-40.27)], alanine aminotransferase (ALT) [23.28\% (19.9227.01)], and aspartate aminotransferase (AST) [23.41\% (18.84-28.70)]. Furthermore, the relative risk of these abnormalities was higher in the patients with severe COVID-19 when compared to non-severe disease [Hypoalbuminemia-2.65 (1.38-5.07); GGT-2.31 (1.6-3.33); ALT-1.76 (1.44-2.15); AST-2.30 (1.82-2.90)]. The SMD of hypoalbuminemia, GGT, ALT, and AST elevation in severe as compared to non-severe were -1.05 ( -1.27 to -0.83$), 0.76(0.40-1.12), 0.42$ $(0.27-0.56)$, and $0.69(0.52-0.86)$, respectively. The pooled prevalence and RR of chronic liver disease as a comorbidity was $2.64 \%(1.73-4)$ and $1.69(1.05-2.73)$ respectively.
\end{abstract}

M. Praveen Kumar and Shubhra Mishra have contributed equally to this work.

Electronic supplementary material The online version of this article (https://doi.org/10.1007/s12072-020-10071-9) contains supplementary material, which is available to authorized users.

Vishal Sharma

docvishalsharma@gmail.com

Praveen Kumar-M

praveenkumarpgiindia@gmail.com

Shubhra Mishra

shubhra.mishra91@gmail.com

Daya Krishna Jha

dayakrishna.jha@gmail.com

Jayendra Shukla

jayendra.shuk11986@gmail.com

Arup Choudhury

drarupc@gmail.com

Ritin Mohindra

ritin.mohindra@gmail.com
Harshal S. Mandavdhare

hmandavdhare760@gmail.com

Usha Dutta

ushadutta@gmail.com

1 Department of Pharmacology, Postgraduate Institute of Medical Education and Research, Chandigarh, India

2 Department of Gastroenterology, Postgraduate Institute of Medical Education and Research, Chandigarh, India

3 Department of Internal Medicine, Postgraduate Institute of Medical Education and Research, Chandigarh, India 
Conclusion The most frequent abnormality in liver functions was hypoalbuminemia followed by derangements in gammaglutamyl transferase and aminotransferases, and these abnormalities were more frequent in severe disease. The systematic review was, however, limited by heterogeneity in definitions of severity and liver function derangements.

\section{Graphic abstract}

Graphical depiction of the summary of meta-analytic findings a) pooled prevalence of abnormalities b) Risk ratio of abnormality in severe versus non-severe COVID-19 c) standardized mean difference (SMD) between severe and non-severe group and d) pooled prevalence for parameters based on severity stratification for bilirubin, alanine aminotransferase (ALT), aspartate aminotransferase (AST), alkaline phosphatase (ALP), gamma-glutamyl transferase (GGT), albumin, globulin and acute hepatic injury (AHI) . Also estimates for overall/total liver disease (TLD) and chronic liver disease (CLD) amongst COVID-19 patients are depicted in a, b, d. For d) In addition to severity stratification, Overall (all studies for a particular estimate) and combined (only those studies which reported severity) estimates are provided.
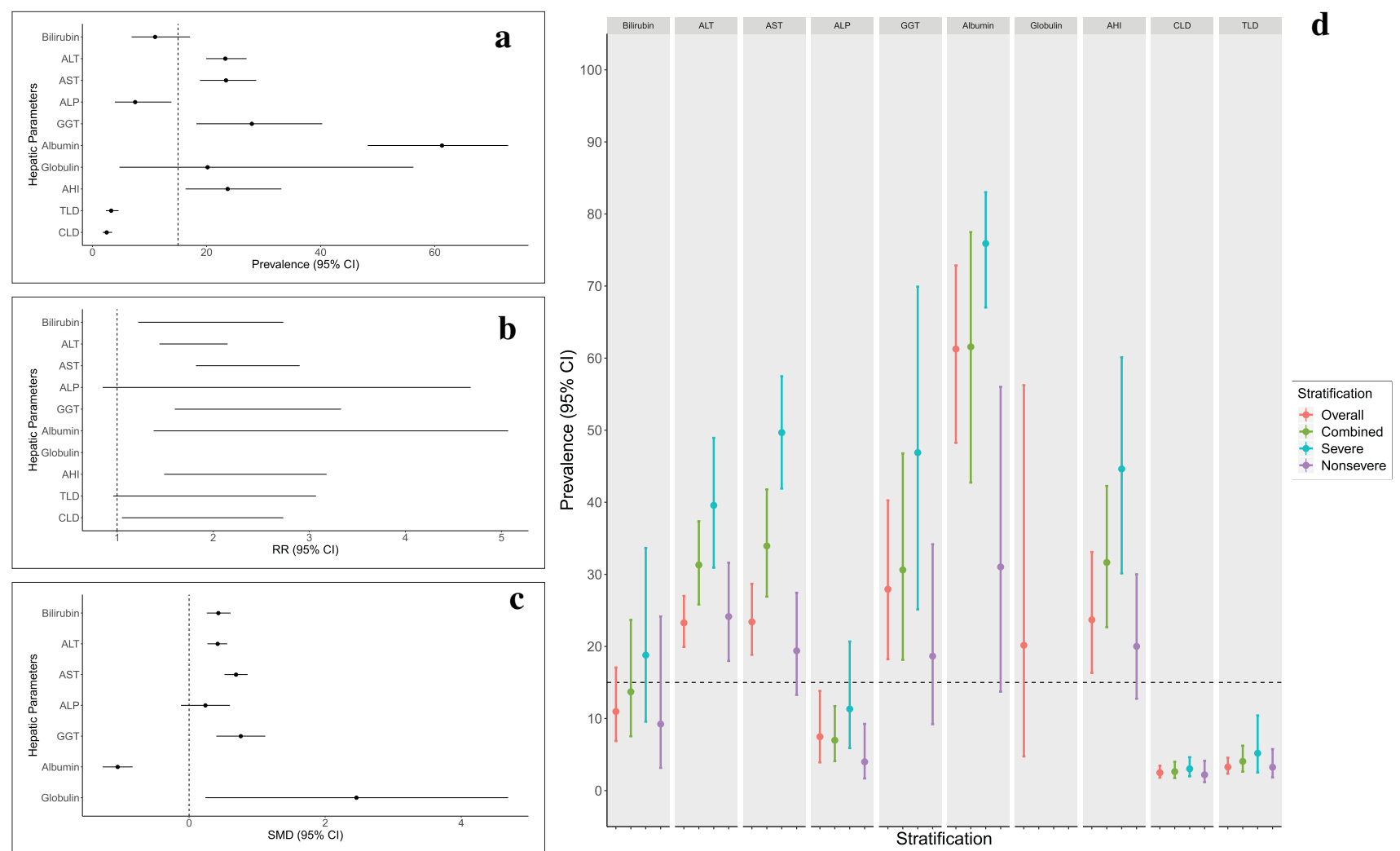

Keywords SARS-CoV-2 · COVID-19 · Cirrhosis · Histology · Transplantation

\section{Introduction}

Coronavirus disease (COVID-19) was first brought to light with the appearance of cases of viral pneumonia in December 2019 in Wuhan city of the Chinese Hubei province [1]. Since then, the disease has spread globally and is recognized as a global pandemic by the World Health Organization (WHO). The disease, caused by severe acute respiratory syndrome coronavirus 2 (SARS-CoV-2), has been responsible for large numbers of hospital admissions and mortality, resulting in a severe stress on health care resources. With time, the understanding of the disease has also improved and it has become apparent that it involves not just the pulmonary system but also the gastrointestinal system, heart, and the liver [2].

The hepatic involvement has been well recognized in the two recent pathogenic coronaviruses, i.e., SARS-COV and Middle East Respiratory Syndrome Coronavirus (MERSCOV). These two viruses had striking genetic similarity (especially SARS-COV) with the novel coronavirus, i.e., SARS-CoV-2 and, therefore, hepatic involvement in this is not entirely unexpected [3]. Indeed, multiple reports have suggested that elevation of liver transaminases does occur 
with the infection of SARS-CoV-2. The purported mechanisms include the possibility of direct effect of the virus on hepatocytes or biliary epithelium, liver injury related to accentuated immune response (cytokine storm) and immune mediated damage, drug toxicity (because of drugs like acetaminophen, antivirals and hydroxychloroquine), and ischemic hepatitis which could occur in patients having multiorgan dysfunction including hemodynamic instability [4]. The biliary epithelium expresses the angiotensin-converting enzyme (ACE-2) receptor which is the known binding site of SARS-CoV-2, while the expression in hepatocytes is possibly much lower. However, the receptor expression has been shown to be upregulated in animals' models of liver injury [4].

The literature regarding hepatic involvement in COVID19 is heterogenous with variability in the definitions of liver dysfunction and differences in the clinical presentation and disease severity of patients included in the published reports $[5,6]$. Therefore, we planned to systematically study the occurrence of liver injury in COVID-19 and also determine the frequency of liver involvement in COVID-19. We also planned to identify any differences in frequency of liver dysfunction with varying disease severity, and identify differences in frequency of liver dysfunction in COVID-19 vis-avis non-COVID disease and also the frequency of underlying liver as a comorbidity in COVID-19 disease.

\section{Materials and methods}

We conducted this systematic review and meta-analysis as per the guidance provided by the PRISMA statement [7].

\section{Search and study selection}

We searched PubMed and Embase on 5th April 2019 for publications using the keywords "COVID", "Novel coronavirus" or "SARS-CoV-2" for all publications after 1st December 2019. No other restrictions for language or ethnicity or type of papers were used. The detailed search strategy is shown in Supplementary Table 1. The results obtained from the two databases were then combined and duplicates removed. Two reviewers (PKM, SM) separately did a title and abstract screening to select any studies reporting on data about underlying hepatic comorbidities or liver dysfunction. The studies selected for full-text screening were seen by two authors (SM and VS) for data extraction. Three other authors checked the data for accuracy and completeness (DKJ, JS, and $\mathrm{AC}$ ). The bibliography of selected papers and relevant reviews were also sought to identify any additional eligible papers and an additional search was done using the original search terms AND "Liver" (both databases) to identify any additional papers as on 23rd April 2020. The detailed flow of the study selection is shown using the PRISMA flowchart (Fig. 1).

\section{Selection of studies}

Two investigators (VS and SM) separately determined if the studies were eligible for inclusion in the meta-analysis and the disagreements were discussed with the third author (PKM) for final decision.

We included studies (1) reporting on frequency of various liver function abnormalities including serum bilirubin, alanine aminotransferases (ALT), aspartate aminotransferase (AST), alkaline phosphatase (ALP), or gamma-glutamyl transferase (GGT) in human patients infected with SARSCoV-2, (2) reporting their median/mean levels for severe and non-severe disease or (3) reporting the frequency of underlying liver disease as a comorbidity in patients with COVID-19.

These studies were included irrespective of age, gender, ethnicity of the reported population, the study designs (cohort, case series, randomized trials), and the language of publication. We excluded the studies (1) if number of patients were $<5$, (2) if studies did not have confirmed cases of COVID-19, (3) if none of the relevant liver functions or data on liver disease as a comorbidity were reported, and (4) study designs like comments, editorials, reviews and systematic reviews which did not provide primary data. These studies were, however, looked at to identify additional papers from bibliography.

\section{Study groups and analysis plan}

We planned to find the pooled prevalence of liver function abnormalities in cases of COVID-19 and compare the frequencies of liver derangements between severe and non-severe COVID-19. We also planned to compare the frequencies of liver derangements between COVID cases and non-COVID cases if such data were available. We also planned to determine the pooled prevalence of underlying liver disease in patients with COVID-19 and compare if this frequency was different between severe and non-severe disease and between COVID and non-COVID disease. Additionally, we planned to compare the standard mean difference between severe and non-severe COVID and COVID and non-COVID cases.

\section{Data extraction}

We extracted data as per: authors, location of study, type of study, type of included patients, number of included patients, frequency of various hepatic derangements (elevation of serum bilirubin, ALT, AST, GGT, ALP, serum albumin) in patients with COVID-19 and separately for 


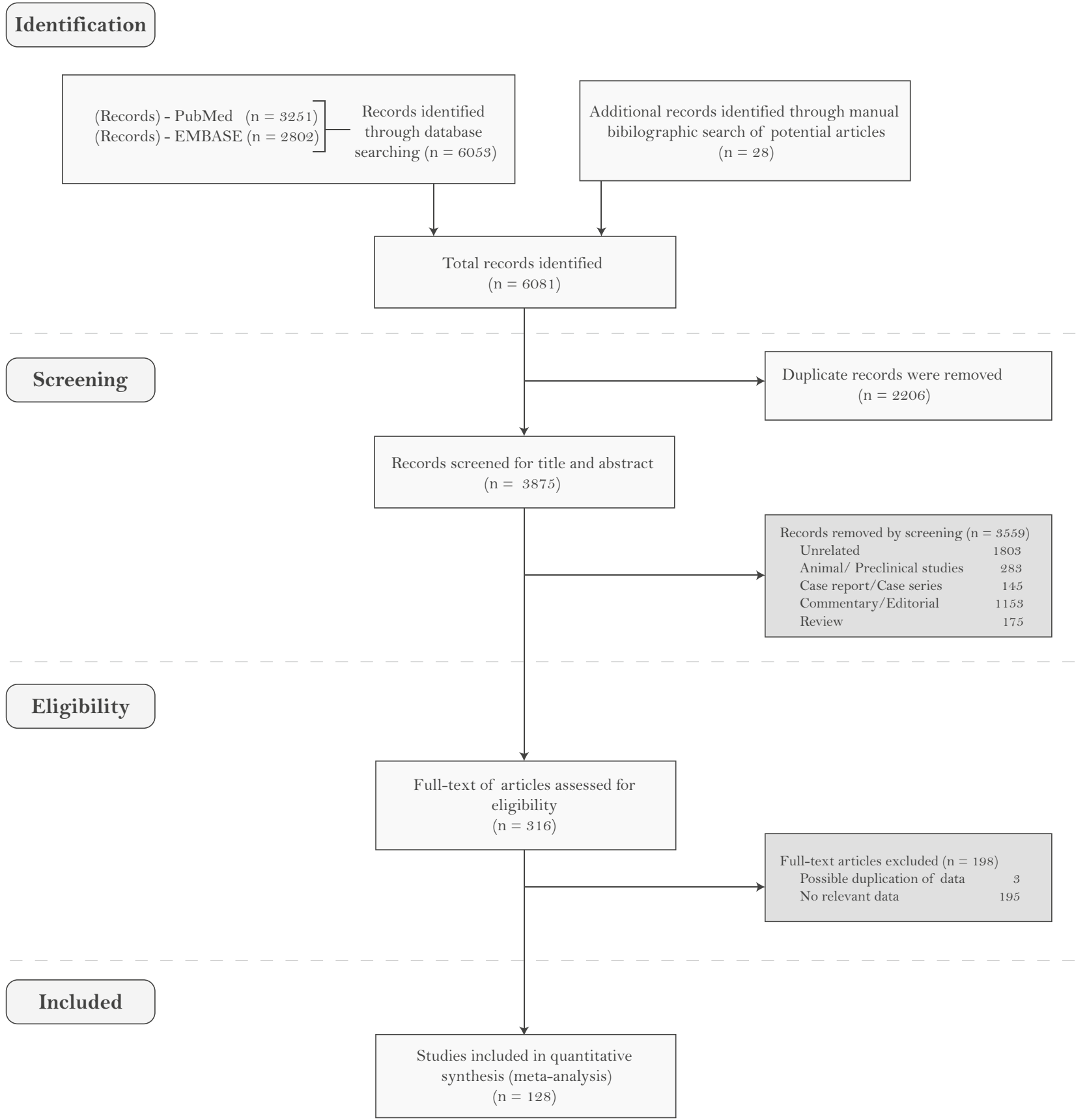

Fig. 1 PRISMA chart showing the flow of study inclusion for the meta-analysis

those with severe and non-severe disease, mean/median values with standard deviation/interquartile range/range of these parameters from studies which compared severe and non-severe disease and also COVID and non-COVID cases. The details regarding the definition of disease severity in each study and details of definition of acute hepatic injury (or hepatic dysfunction) were also extracted.

\section{Statistical analysis}

The statistical analysis was performed using $\mathrm{R}$ statistical software (version 3.6.1) [8]. In addition to the base package, meta, readxl, and ggplot2 packages were used [9-11]. The number of events were summarized as events per 100 observations. The inverse variance method with logit 
transformation along with Clopper Pearson confidence interval for individual studies was used for the meta-analytic pooled prevalence in the overall and subgroup population. For difference in prevalence between subgroups, the inverse variance method was used for summarizing. A continuity correction of 0.5 was applied for studies with zero cell frequency. For continuous variable standardized mean differences (SMD) were computed between the evaluated subgroups and inverse variance method was used for summarizing. Hedges's g correction was used for bias correction of standardized mean differences. For studies reporting mean and standard deviation, we directly took the reported values for analysis. For studies reporting median and interquartile range or median and range, the mean and standard deviation were computed from the reported values based on Luo 2018 and Wan 2014 methodology [12, 13]. For studies reporting only mean along with $p$ value, the t statistics were computed, which was then used for calculation of standard deviation. For studies reporting individual data, we computed mean and SD from the data. Heterogeneity of prevalence, RR, and SMD were tested with $p$ value of heterogeneity $(p<0.10)$ and $I^{2}$ was computed. $I^{2}$ value was taken for reporting for heterogeneity as, in addition to quantifying heterogeneity, it also assesses the impact of heterogeneity on the metaanalysis. Random effects models were used for summarizing results irrespective of heterogeneity. For prevalence, 95\% C.I of pooled prevalence was reported. For studies comparing prevalence and SMD between two groups, $p$ value of $<0.05$ was considered statistically significant and $95 \%$ confidence intervals (CIs) were reported. The forest plots were constructed for visualization of results.

\section{Results}

\section{Search results and included studies}

The search yielded a total of 6053 citations and additional 28 papers were identified from other sources like bibliography search. After removal of 2206 duplicates, 3875 papers were screened for title and abstract. After removal of 3559 papers for various reasons (Fig. 1), a total of 316 papers were eligible for full-text screening. Eventually a total of 128 papers were included in at least one of the different analyses which were conducted as part of the meta-analysis (Supplementary Table 2).

\section{Overall frequency of various abnormalities in liver function in COVID-19}

The pooled prevalence of hyperbilirubinemia reported in various studies was $10.98 \%$ (95\% CI 6.87-17.08; $I^{2} 94 \%$ ) (Fig. 2a). The pooled prevalence of alanine aminotransferase
(ALT) and aspartate aminotransferase (AST) elevation reported in various studies was $23.28 \%$ (19.92-27.01; 90\%) and $23.41 \%$ (18.84-28.70; 95\%), respectively (Figs. 3a and $4 \mathrm{a})$. The pooled prevalence of ALP and GGT elevation among overall reported studies was 7.48\% (3.91-13.83; $75 \%$ ) and $27.94 \%$ (18.22-40.27; 93\%), respectively (Supplementary Fig. 1 and Fig. 5A). The pooled prevalence of hypoalbuminemia among overall reported studies was $61.27 \%$ (48.24-72.87; 91\%) (Fig. 6a). The overall pooled frequency of increased globulin levels reported was $20.17 \%$ (4.73-56.25; 95\%) (Supplementary Fig. 2). The pooled frequency of acute hepatic injury as reported in various studies is shown in Supplementary Fig. 3. The pooled prevalence of hepatitis B, fatty liver, total liver disease, and chronic liver disease as a comorbidity are shown in Supplementary Figs. 4-7. The pooled prevalence of chronic liver disease as a comorbidity in various studies was $2.49 \%(1.79-3.46$, 84\%) (Supplementary Fig. 7). The pooled frequency of hepatitis B, non-alcoholic fatty liver disease, and total frequency of any liver disease are shown in Supplementary Figs. 4, 5 and 6.

\section{Comparison between severe and non-severe COVID-19}

The pooled prevalence of bilirubin abnormality among the studies which reported the finding on the basis of underlying severity of COVID-19 was $13.71 \%$ (95\% CI 7.53-23.69; $\left.I^{2} 95 \%\right)$. The pooled frequency of hyperbilirubinemia in severe COVID disease was $18.80 \%(9.55-33.67$; $91 \%)$, while in non-severe disease, it was 9.24\% (3.15-24.17; 97\%) (Fig. 2b). The risk ratio (RR) of deranged bilirubin concentration in severe as compared to non-severe subgroups was 1.82 (95\% CI 1.22-2.73; $I^{2}$ 66\%) (Fig. 2c).The SMD for bilirubin concentration between the severe and non-severe group was 0.43 (95\% CI 0.26-0.61; $I^{2} 66 \%$ ) (Fig. 2d).

The pooled prevalence of ALT and AST abnormality among the studies reporting severity was $31.31 \%$ (25.82-37.37; 87\%) (Fig. 3b) and 33.95\% (26.90-41.79; 91\%) (Fig. 4b). The pooled frequency of ALT elevation was $39.58 \%$ (30.92-48.94; $80 \%$ ) for severe and $24.15 \%$ (17.98-31.63; 90\%) for non-severe disease (Fig. 3c). The pooled frequency of AST elevation was $49.68 \%$ $(41.90-57.49 ; 73 \%)$ and $19.40 \%(13.27-27.45 ; 91 \%)$, respectively, for severe and non-severe disease (Fig. 4c). The RR of SGPT and SGOT abnormality in the severe as compared to non-severe subgroups were $1.76(1.44-2.15 ; 65 \%)$ and $2.30(1.82-2.90,67 \%)$, respectively (Figs. $3 \mathrm{~d}$ and $4 \mathrm{~d}$ ). The SMD for SGPT and SGOT concentration between the severe and non-severe groups were $0.42(0.27-0.56 ; 70 \%)$ and $0.69(0.52-0.86 ; 76 \%)$, respectively (Figs. $3 \mathrm{e}$ and $4 \mathrm{e})$.

The pooled prevalence of ALP and GGT abnormality among the studies reporting severity was $6.99 \%$ 
$\mathbf{a}$

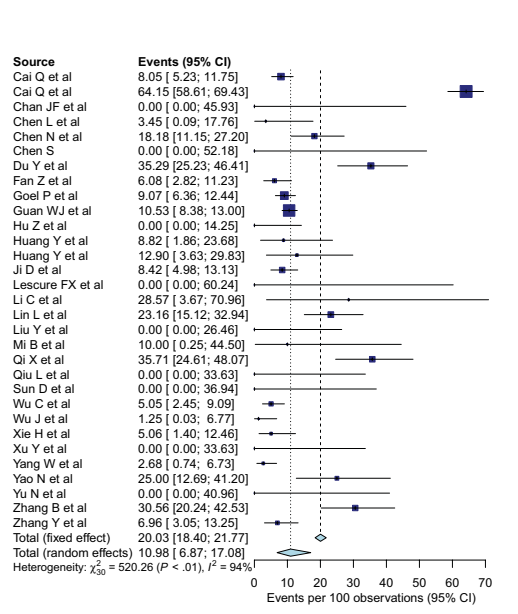

b

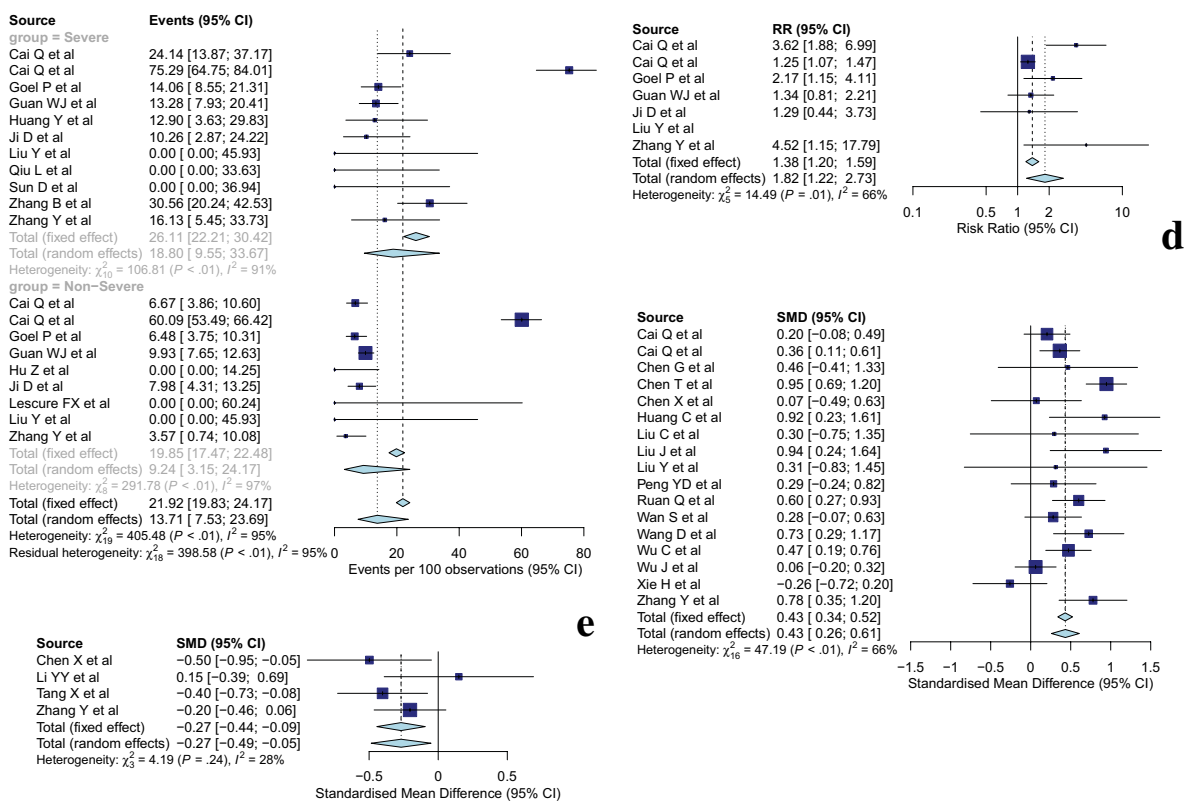

Fig. 2 Forest plots of bilirubin. Random effects summary were reported in the manuscript. The heterogeneity was measured across studies by $I^{2}$ and $p$ value of heterogeneity. a Pooled prevalence of hyperbilirubinemia reported in various studies. b Pooled prevalence of hyperbilirubinemia among the studies which reported the findings on the basis of underlying severity of COVID-19. $c$ Risk ratio (RR) of hyperbilirubinemia in severe as compared to non-severe subgroups.
For RR, the middle vertical line at 1 stands for line of no-difference d SMD for bilirubin concentration between the severe and non-severe groups. For SMD, the middle vertical line at 0 stands for line of no difference. e SMD of bilirubin concentration between COVID and non-COVID subgroups. $S M D$ standardized mean difference, $R R$ risk ratio, $C I$ confidence interval
(4.08-11.72; 59\%) (Supplementary Fig. 1b) and 30.62\% (18.13-46.79; 94\%) (Fig. 5b) with subdivision for ALP being $11.33 \%(5.89-20.69 ; 42 \%)$ for severe and $4 \%$ (1.68-9.26; 64\%) for non-severe and for GGT being $46.90 \%(25.13-69.92 ; 89 \%)$ and $18.66 \%$ (9.20-34.18; 93\%). The RR of ALP and GGT abnormality in severe as compared to non-severe subgroups were $1.99(0.85-4.68$; 22\%) (Supplementary Fig. 1c) and 2.31 (1.6-3.33; 55\%) (Fig. 5c), respectively. The SMD for ALP and GGT concentration between the severe and non-severe groups were 0.24 ( -0.12 to $0.60 ; 83 \%)$ (Supplementary Fig. 1d) and $0.76(0.40-1.12 ; 82 \%)$ (Fig. 5d), respectively.

The pooled prevalence of albumin abnormality among the studies reporting severity was $61.57 \%(42.73-77.48$; $90 \%)$ with subdivision of $75.91 \%(67.02-83.02 ; 35 \%)$ for severe and $31.04 \%(13.72-56.02 ; 84 \%)$ for non-severe (Fig. 6b). The RR of albumin abnormality in severe as compared to non-severe subgroup was 2.65 (1.38-5.07; 79\%) (Fig. 6c). The SMD for albumin concentration between the severe and non-severe groups was -1.05 ( -1.27 to $-0.83 ; 77 \%$ ) (Fig. 6d). The SMD for globulin concentration between severe and non-severe groups was $2.46(0.24-4.69,99 \%)$ (Supplementary Fig. 2b).
The pooled prevalence of chronic liver disease (CLD) among studies reporting severity was $2.64 \%$ (1.73-4; $72 \%)$ with $3.03(1.97-4.64 ; 21 \%)$ among severe and $2.20 \%$ (1.16-4.15; 83\%) among non-severe (Supplementary Fig. 7b). The RR of CLD in severe as compared to nonsevere subgroup was $1.69(1.05-2.73 ; 0 \%)$ (Supplementary Fig. 7c).

\section{Presence of acute hepatic injury (supplementary Fig. 3)}

The pooled prevalence of acute hepatic injury among overall reported studies was $23.70 \%$ (95\% CI 16.31-33.11; $I^{2}$ 97\%) (Supplementary Fig. 3a). Among the studies reporting severity, the total frequency of acute hepatic injury was $31.66 \%$ (22.66-42.27; 91\%), whereas in severe disease, it was $44.63 \%$ (30.13-60.11; 88\%), and in non-severe disease, it was $20.02 \%$ (12.74-30.02; 88\%) (Supplementary Fig. 3b). The RR of acute hepatic injury in severe as compared to non-severe was $2.18(1.49-3.18,67 \%)$ (Supplementary Fig. 3c). 
$\mathbf{a}$

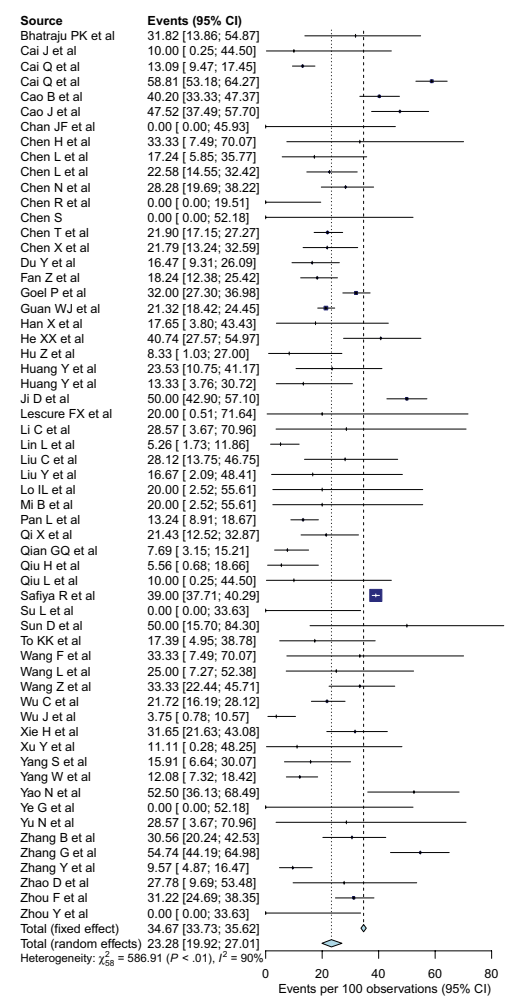

b
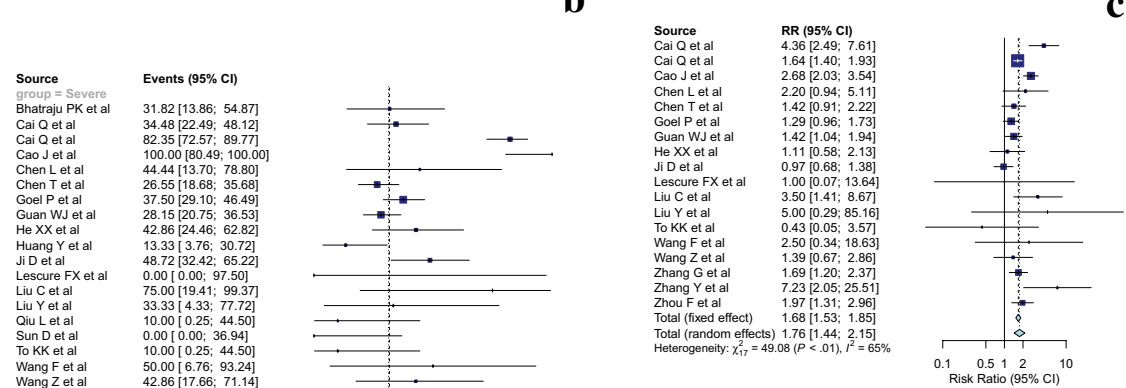

d

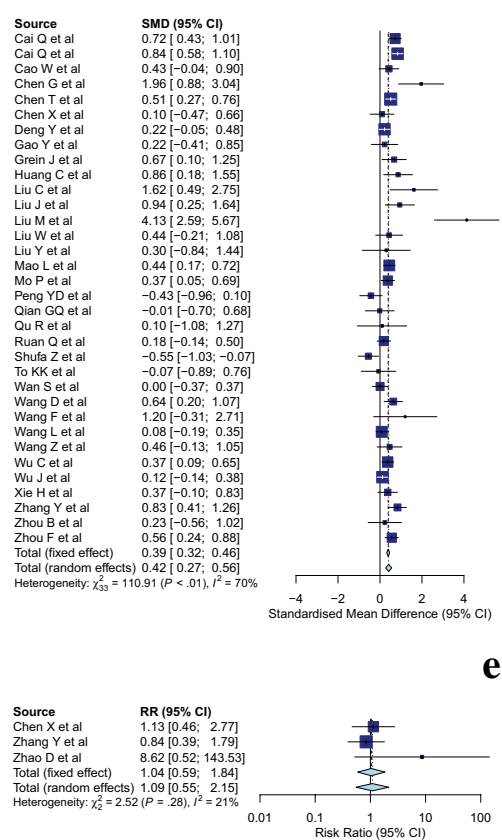

Fig. 3 Forest plots of alanine aminotransferase (ALT). Random effects summary were reported in the manuscript. The heterogeneity was measured across studies by $I^{2}$ and $p$ values of heterogeneity. a Pooled prevalence of ALT elevation reported in various studies. b Pooled prevalence of ALT elevation among the studies which reported the finding on the basis of underlying severity of COVID19. $\mathbf{c}$ Risk ratio (RR) of ALT elevation in severe as compared to non-

\section{Comparison between COVID and non-COVID disease}

The RR of ALT and AST abnormality in the COVID as compared to non-COVID subgroups were 1.09 (95\% CI $\left.0.55-2.15 ; I^{2} 21 \%\right)$ and $1.02(0.45-2.30 ; 48 \%)$, both statistically non-significant (Figs. 2e and 3e). The SMD for SGPT and SGOT concentration between the COVID and non-COVID group were $0.18(0.05-0.32 ; 0 \%)$ and 0.02 ( -0.47 to 0.52 ; $91 \%$ ) (Figs. $2 \mathrm{f}$ and $3 \mathrm{f}$ ). The SMD of bilirubin, GGT, and albumin concentration between COVID and non-COVID subgroups were -0.27 ( -0.49 to -0.05 , $28 \%), 0.12$ ( -0.24 to $0.48 ; 58 \%)$ and $0.82(0.30-1.33$; $82 \%$ ), respectively (Figs. 2e, 5e, 6e). severe subgroups. For RR, the middle vertical line at 1 stands for line of no-difference. d SMD for ALT elevation between the severe and non-severe groups. For SMD, the middle vertical line at 0 stands for line of no difference. e RR of ALT elevation in the COVID as compared to non-COVID subgroups. f SMD of ALT elevation between COVID and non-COVID subgroup. SMD standardized mean difference, $R R$ risk ratio, $C I$ confidence interval

\section{Hepatic histological findings in COVID-19}

Only limited cases have reported findings on liver histology using either post-mortem histology or liver biopsy in patients with COVID-19 and are tabulated in Supplementary Table 3 . The findings reported are non-specific and include sinusoidal dilatation, mild activity in portal area, and lobules and occasional necrosis. It is unclear if these are a manifestation of virus mediated liver injury or due to other factors like drugs or immune injury. 
a

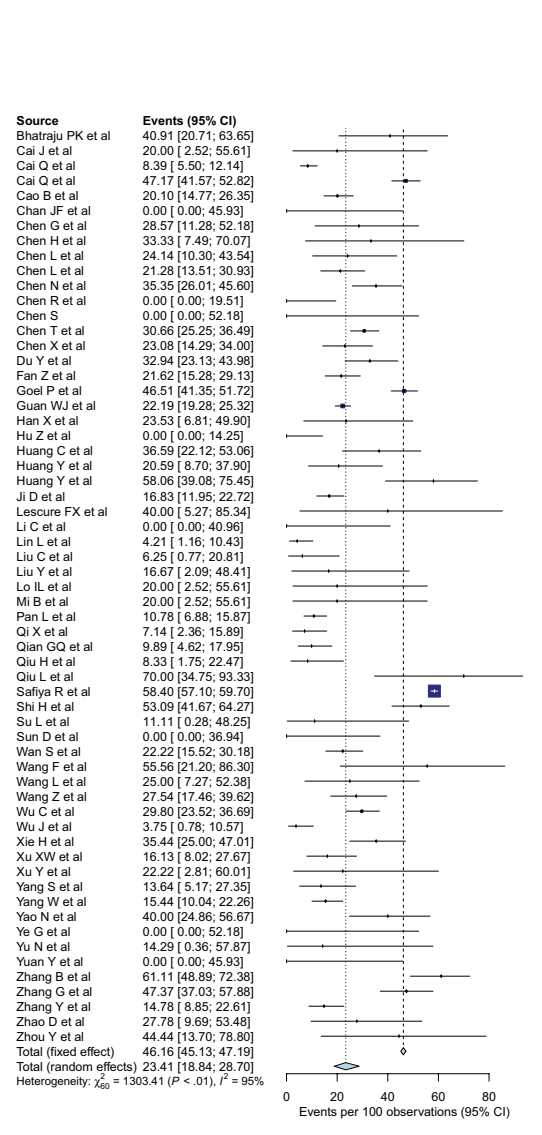

b

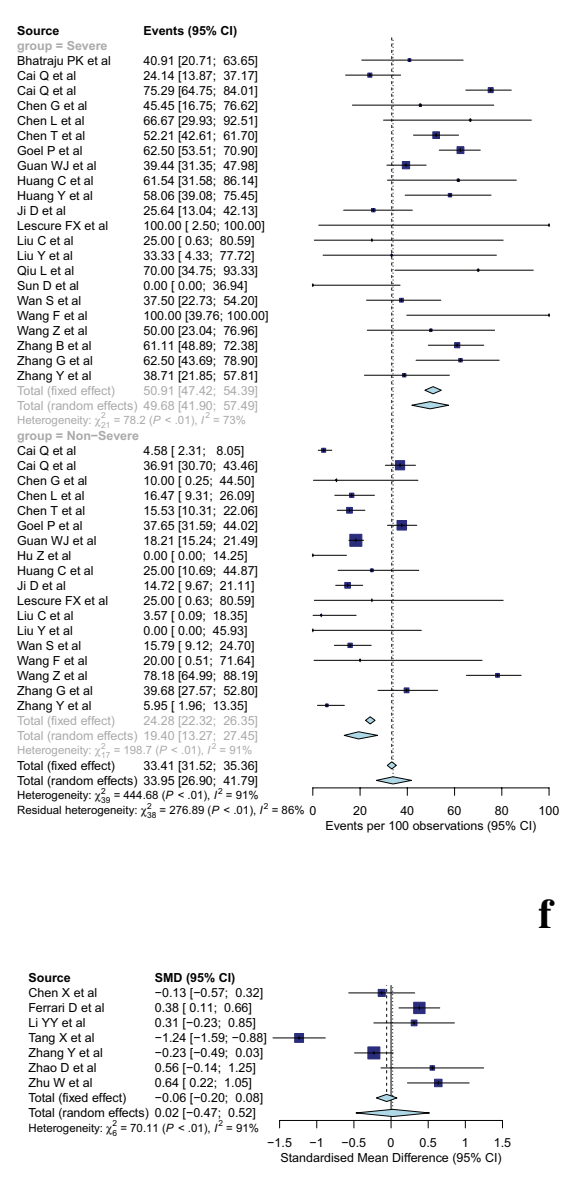

c

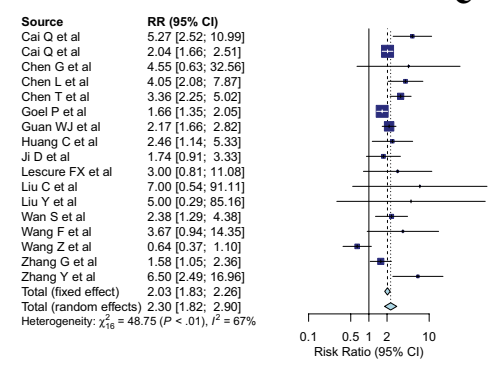

d

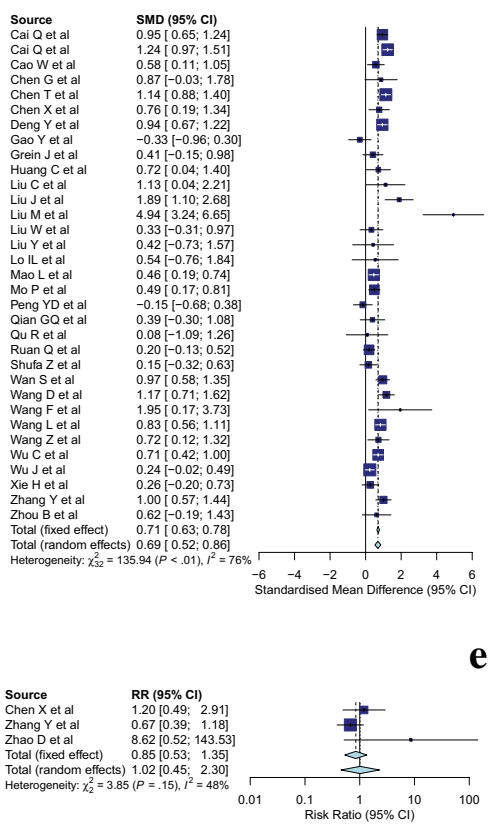

Fig. 4 Forest plots of aspartate aminotransferase (AST). Random effects summary were reported in the manuscript. The heterogeneity was measured across studies by $I^{2}$ and $p$ values of heterogeneity. a Pooled prevalence of AST elevation reported in various studies. b Pooled prevalence of AST elevation among the studies which reported the finding on the basis of underlying severity of COVID19. $\mathbf{c}$ Risk ratio (RR) of AST elevation in severe as compared to non-

\section{COVID-19 in liver transplant recipients}

Some case reports and studies have reported the outcomes of COVID-19 in patients with liver transplant and are shown in Supplementary Table 4.

\section{Discussion}

In this systematic review, we found that the derangements of liver functions were frequently noted in patients with COVID-19. The most frequent abnormality noted was hypoalbuminemia followed by elevations of gamma-glutamyl transferase, aminotransferases, bilirubin, and alkaline severe subgroups. For RR, the middle vertical line at 1 stands for line of no-difference. d SMD for AST elevation between the severe and non-severe groups. For SMD, the middle vertical line at 0 stands for line of no difference. e RR of AST elevation in the COVID as compared to non-COVID subgroups. $\mathbf{f}$ SMD of AST elevation between COVID and non-COVID subgroups. SMD standardized mean difference, $R R$ risk ratio, $C I$ confidence interval

phosphatase. Furthermore, when comparison of severe with non-severe COVID-19 cases was done, liver function abnormalities like hypoalbuminemia, GGT, and aminotransferase and bilirubin elevations were more frequent in those with severe disease. However, serum alkaline phosphatase elevations were not significantly higher in the severe group of patients. The pooled frequency of elevation of ALT and AST was similar in the overall COVID cases, but, interestingly, the prevalence of AST elevations was more than ALT in the severe COVID disease.

The most frequent abnormality noted in our meta-analysis was hypoalbuminemia. This is possibly related to the fact that albumin is a negative acute phase reactant rather than a manifestation of a hepatic synthetic dysfunction. Our 


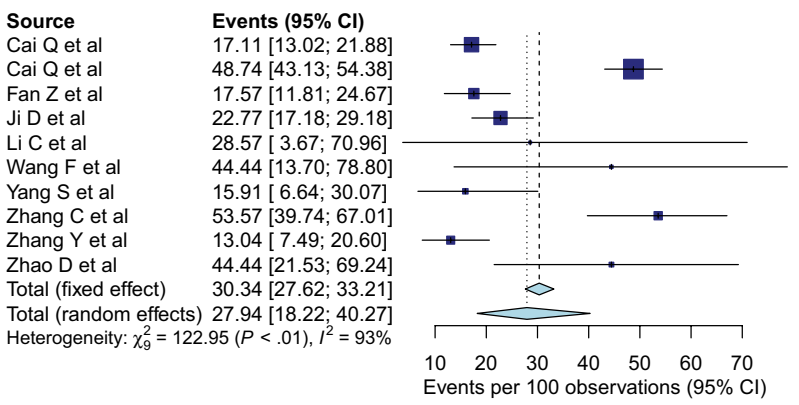

b

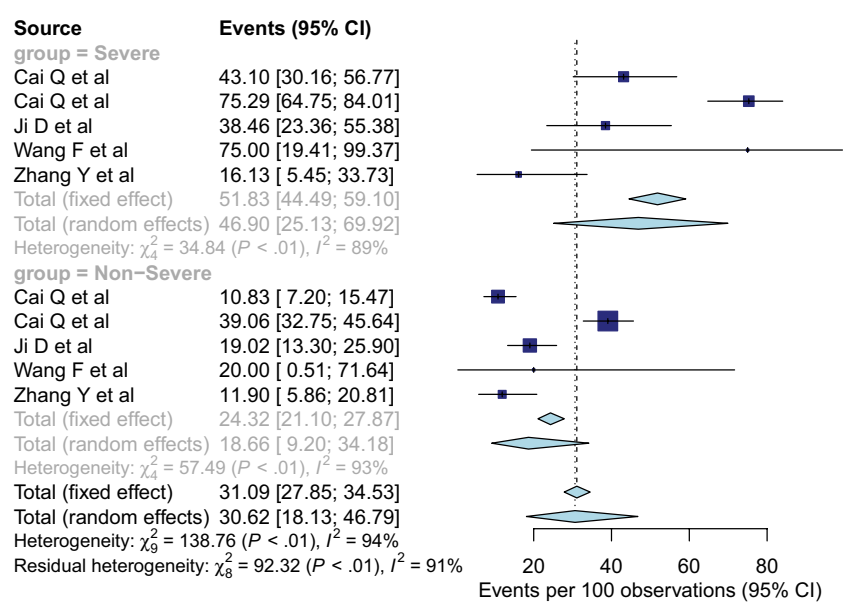

Fig. 5 Forest plots of gamma-glutamyl transferase (GGT). Random effects summary were reported in the manuscript. The heterogeneity was measured across studies by $I^{2}$ and $p$ values of heterogeneity. a Pooled prevalence of GGT elevation reported in various studies. b Pooled prevalence of GGT elevation among the studies which reported the finding on the basis of underlying severity of COVID-19. c Risk ratio (RR) of GGT elevation in severe as compared to non-

meta-analysis confirms that the liver enzyme elevations in COVID-19, even in the severe COVID category, are mildto-moderate in most of the cases. Although there are a few reports of aminotransferase elevation to a high degree $(>10$ times of upper limit), this is an uncommon phenomenon $[14,15]$. Furthermore, liver failure is exceedingly rare with only a single case report available in a patient who had severe COVID associated with use of multiple drugs and progression of liver function derangement after admission [14]. As of now, it is not possible to conclusively ascribe these elevations to be related to direct viral injury. Most of the studies included in our analysis reported use of multiple drugs like antibiotics, antivirals (lopinavir/ritonavir combination, arbidol, oseltamivir, favipiravir, remdesvir, hydroxychloroquine), and steroids. Many of these drugs

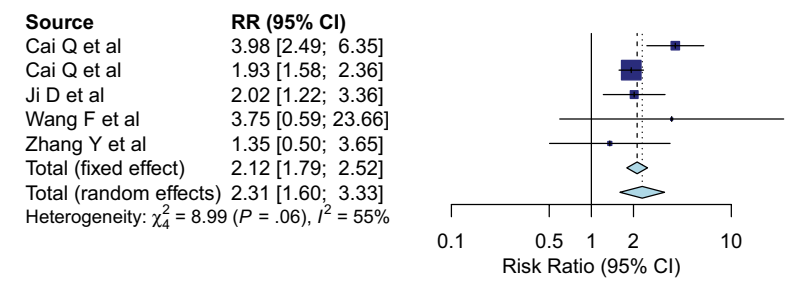

d

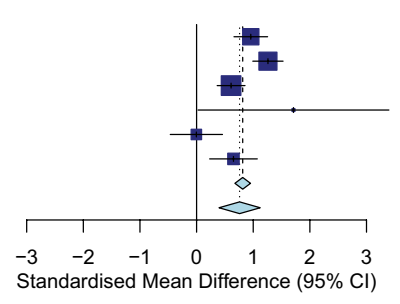

$\begin{array}{ll}\text { Source } & \text { SMD }(95 \% \mathrm{Cl}) \\ \text { Cai Q et al } & 0.96[0.66 ; 1.25] \\ \text { Cai Q et al } & 1.26[0.99 ; 1.53] \\ \text { Chen T et al } & 0.61[0.36 ; 0.85] \\ \text { Wang F et al } & 1.71[0.03 ; 3.40] \\ \text { Xie H et al } & -0.00[-0.46 ; 0.46] \\ \text { Zhang Y et al } & 0.65[0.23 ; 1.07] \\ \text { Total (fixed effect) } & 0.81[0.68 ; 0.95] \\ \text { Total (random effects) } & 0.76[0.40 ; 1.12] \\ \text { Heterogeneity: } \chi_{5}^{2}=28.00(P<.01), I^{2}=82 \%\end{array}$

Heterogeneity: $\chi_{5}^{2}=28.00(P<.01), I^{2}=82 \%$ $\begin{array}{lllllll}-3 & -2 & -1 & 0 & 1 & 2 & 3 \\ \text { Standardised Mean Difference } & (95 \% & \mathrm{Cl})\end{array}$

e

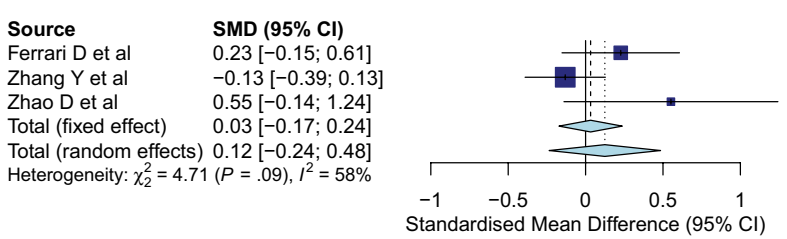

severe subgroups. For RR, the middle vertical line at 1 stands for line of no-difference. d SMD for GGT elevation between the severe and non-severe groups. For SMD, the middle vertical line at 0 stands for line of no difference. e SMD of GGT elevation between COVID and non-COVID subgroup. $S M D$ standardized mean difference, $R R$ risk ratio, $C I$ confidence interval

could have contributed to occurrence of liver dysfunction after hospitalization. Furthermore, ischemia and immune mediated injury due to cytokine storm could be responsible for liver dysfunction in patients with severe COVID disease.

SARS-CoV-2 uses the ACE- 2 receptor for gaining entry into the cells including the type 2 alveolar epithelial cells in the lungs. Chai et al. analyzed the expression of ACE-2 receptors in liver tissue and found that their expression on cholangiocytes $(59.7 \%)$ was much stronger than on hepatocytes (2.6\%) [16]. It has also been observed that ACE-2 expression increases in hepatocytes, in cases of liver injury $[4,17]$. At least, one report suggests that viral RNA could be detected in liver tissue raising the possibility of viral mediated liver injury [18]. On the other hand, Chu et al. demonstrated a significant replication of SARS-CoV-2 on 
a

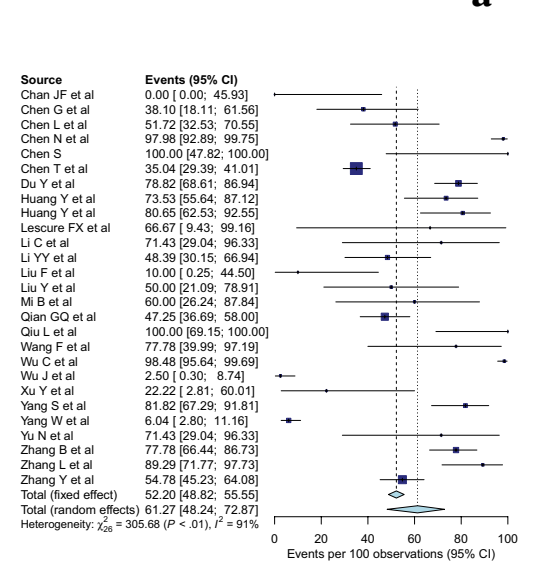

b

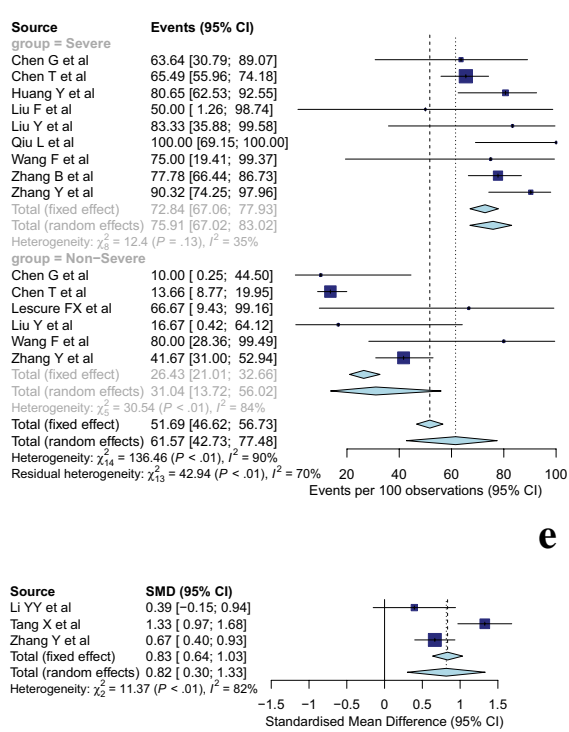

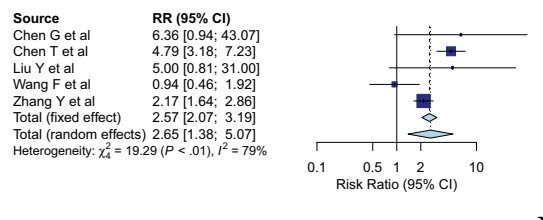

d

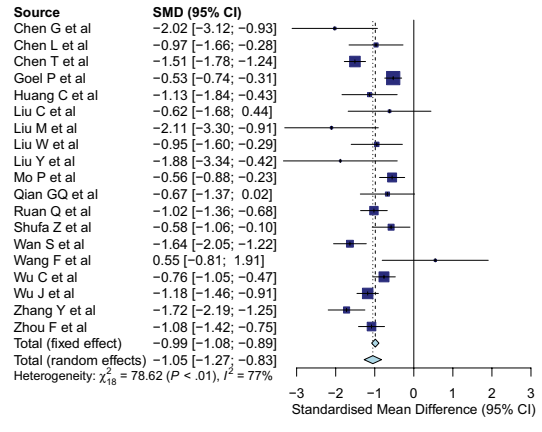

Fig. 6 Forest plots of albumin. Random effects summary were reported in the manuscript. The heterogeneity was measured across studies by $I^{2}$ and $p$ values of heterogeneity. a Pooled prevalence of hypoalbuminemia reported in various studies. b Pooled prevalence of hypoalbuminemia among the studies which reported the finding on the basis of underlying severity of COVID-19. c Risk ratio (RR) of hypoalbuminemia in severe as compared to non-severe subgroups.
For RR, the middle vertical line at 1 stands for line of no-difference. d SMD for hypoalbuminemia between the severe and non-severe groups. For SMD, the middle vertical line at 0 stands for line of no difference. e SMD of hypoalbuminemia between COVID and nonCOVID subgroups. $S M D$ standardized mean difference, $R R$ risk ratio, CI confidence interval

patients with COVID-19. However, the frequency of underlying liver disease was statistically not different between severe and non-severe diseases. Unfortunately, our metaanalysis cannot directly provide evidence regarding the effect of underlying liver disease on outcomes of disease or whether it predisposes to severe COVID illness. Furthermore, the group 'total liver disease' is also a heterogeneous group comprising chronic hepatitis B, non-alcoholic fatty liver disease, and chronic liver disease. Therefore, we also did additional analysis of the frequency of chronic liver disease and underlying hepatitis B separately, and the findings regarding frequency of underlying liver disease in severe and non-severe COVID did not seem to change except that the relative risk of underlying chronic liver disease was more in those with severe COVID disease. The effect of COVID on patients with previous transplant has been reported infrequently and data are still unclear if these patients are at an increased risk of severe disease. (Supplementary Table 4).

There are some limitations to the current systematic review and meta-analysis. The most important concern is the variability in the definitions of liver dysfunction or acute liver injury (Supplementary Table 5), differences in the normal values of liver enzymes in various studies, and heterogeneity in definition of severe and non-severe COVID groups, while most studies defined severe disease as per WHO criteria, many others have defined severe 
disease as ICU admissions, non-survivors, etc. (Supplementary Table 6). This adds significant heterogeneity, because a study only on ICU patients (which compares survivors and non-survivors) is likely to have almost all cases qualifying to the WHO definition of severity [1]. Similarly, although we compared the liver dysfunction in COVID and non-COVID disease, the analysis was limited by the small number of participating studies and heterogeneity in definition of non-COVID cases in various reports. Furthermore, although some studies indicate that the elevation of liver enzymes is a delayed phenomenon and may occur in the second week of illness, we are not able to clarify if this is because of worsening inflammation as part of disease course, or other factors like multiple drugs used in these patients [21]. Also, most of the included studies are from China, the country of initial impact of COVID19 , and, therefore, may not be representative of differences among various populations. Furthermore, the impact of COVID-19 on outcomes in patients with underlying liver disease is less clear. Some case reports have suggested that SARS-CoV2 could be the cause of acute deterioration in patients with underlying chronic liver disease, resulting in Acute-on-Chronic Liver Failure (ACLF) [22, 23]. However, there are multiple strengths of the meta-analysis, as we have compared multiple parameters of liver function, and for most of these, a fair number of studies were available. Also, the study clarifies the frequency of liver function abnormalities in severe and non-severe diseases. Furthermore, the pooled prevalence of underlying liver disease as a comorbidity has also been clarified.

To conclude, liver function abnormalities, especially hypoalbuminemia, GGT, and aminotransferase elevations, are frequent in patients with COVID-19 disease and the patients with severe disease are more likely to have these liver function derangements.

\footnotetext{
Author contributions PKM: conception, data extraction and validation, analysis, manuscript review, and approval. SM: data extraction, initial draft, manuscript review, and approval. DKJ, JS, and AC: data extraction and validation, manuscript review, and approval. RM, HSM, and UD: important intellectual content, manuscript review, and approval. VS: conception, data extraction, validation, initial draft, important intellectual content, manuscript review, and approval.
}

Funding None.

\section{Compliance with ethical standards}

Conflict of interest M. Praveen Kumar, Shubhra Mishra, Daya Krishna Jha, Jayendra Shukla, Arup Choudhury, Ritin Mohindra, Harshal S. Mandavdhare, Usha Dutta, and Vishal Sharma declare that they have no conflict of interest.

\section{References}

1. World Health Organisation. Interim guidance: Clinical management of severe acute respiratory infection (SARI) when COVID-19 disease is suspected. 2nd Edition. WHO/2019-nCoV/ clinical/2020.4

2. Cheung KS, Hung IF, Chan PP, Lung KC, Tso E, Liu R, et al. Gastrointestinal manifestations of SARS-CoV-2 infection and virus load in fecal samples from the Hong Kong cohort and systematic review and meta-analysis. Gastroenterology 2020. https://doi. org/10.1053/j.gastro.2020.03.065

3. Xu L, Liu J, Lu M, Yang D, Zheng X. Liver injury during highly pathogenic human coronavirus infections. Liver Int 2020. https:// doi.org/10.1111/liv.14435

4. Guan GW, Gao L, Wang JW, Wen XJ, Mao TH, Peng SW, Zhang T, Chen XM, Lu FM. Exploring the mechanism of liver enzyme abnormalities in patients with novel coronavirus-infected pneumonia. Zhonghua Gan Zang Bing Za Zhi 2020;28(2):E002. https ://doi.org/10.3760/cma.j.issn.1007-3418.2020.02.002

5. Bangash MN, Patel J, Parekh D. COVID-19 and the liver: little cause for concern. Lancet Gastroenterol Hepatol 2020. https://doi. org/10.1016/S2468-1253(20)30084-4

6. Zhang C, Shi L, Wang FS. Liver injury in COVID-19: management and challenges. Lancet Gastroenterol Hepatol 2020;5(5):428-430. https://doi.org/10.1016/S2468-1253(20)30057-1

7. Moher D, Liberati A, Tetzlaff J, PRISMA Group. Preferred reporting items for systematic reviews and meta-analyses: the PRISMA statement. Ann Intern Med 2009;151(4):264-269

8. R Core Team (2019). R: A language and environment for statistical computing. R Foundation for Statistical Computing, Vienna, Austria. URL https://www.R-project.org/. Accessed 23 Apr 2020

9. Schwarzer G. meta: an R package for meta-analysis. R News 2007;7(3):40-45

10. Hadley Wickham and Jennifer Bryan (2019). readxl: Read Excel Files. R package version 1.3.1. https://CRAN.R-project.org/packa ge $=$ readxl. Accessed 23 Apr 2020

11. Wickham H. ggplot2: elegant graphics for data analysis. New York: Springer-Verlag; 2016

12. Luo D, Wan X, Liu J, Tong T. Optimally estimating the sample mean from the sample size, median, mid-range, and/or mid-quartile range. Stat Methods Med Res 2018;27(6):1785-805

13. Wan X, Wang W, Liu J, Tong T. Estimating the sample mean and standard deviation from the sample size, median, range and/or interquartile range. BMC Med Res Methodol 2014;14:135

14. Weber S, Mayerle J, Irlbeck M, Gerbes AL. Severe liver failure during SARS-CoV-2 infection. Gut 2020. https://doi.org/10.1136/ gutjnl-2020-321350

15. Wander P, Epstein M, Bernstein D. COVID-19 presenting as acute hepatitis. Am J Gastroenterol 2020. https://doi.org/10.14309/ ajg.0000000000000660

16. Chai X, Hu L, Zhang Y, Han W, Lu Z, Ke A, et al. Specific ACE2 expression in cholangiocytes may cause liver damage after 2019-nCoV infection. bioRxiv 2020. https://doi. org/10.1101/2020.02.03.931766

17. Herath CB, Warner FJ, Lubel JS, Dean RG, Jia Z, Lew RA, et al. Upregulation of hepatic angiotensin-converting enzyme 2 (ACE2) and angiotensin-(1-7) levels in experimental biliary fibrosis. J Hepatol 2007;47(3):387-395

18. Tian S, Xiong Y, Liu H, Niu L, Guo J, Liao M, Xiao SY. Pathological study of the 2019 novel coronavirus disease (COVID-19) through postmortem core biopsies. Mod Pathol 2020. https://doi. org/10.1038/s41379-020-0536-x

19. Chu H, Chan JF, Yuen TT, Shuai H, Yuan S, Wang Y, et al. Comparative tropism, replication kinetics, and cell damage profiling of SARS-CoV-2 and SARS-CoV with implications for clinical 
manifestations, transmissibility, and laboratory studies of COVID19: an observational study. Lancet Microbe 2020. https://doi. org/10.1016/S2666-5247(20)30004-5

20. Yamada J, Tomiyama H, Yambe M, Koji Y, Motobe K, Shiina K, et al. Elevated serum levels of alanine aminotransferase and gamm glutamyltransferase are markers of inflammation and oxidative stress independent of the metabolic syndrome. Atherosclerosis 2006;189(1):198-205

21. Yao N, Wang SN, Lian JQ, Sun YT, Zhang GF, Kang WZ, et al. Clinical characteristics and influencing factors of patients with novel coronavirus pneumonia combined with liver injury in Shaanxi region. Zhonghua Gan Zang Bing Za Zhi 2020;28:E003. https://doi.org/10.3760/cma.j.cn501113-20200226-00070
22. Qiu H, Wander P, Bernstein D, Satapathy SK. Acute on chronic liver failure from novel severe acute respiratory syndrome coronavirus 2 (SARS-CoV-2). Liver Int 2020. https://doi.org/10.1111/ liv. 14506

23. Große K, Kramer M, Trautwein C, Bruns T. SARS-CoV-2 as an extrahepatic precipitator of acute-on-chronic liver failure. Liver Int 2020. https://doi.org/10.1111/liv.14540

Publisher's Note Springer Nature remains neutral with regard to jurisdictional claims in published maps and institutional affiliations. 